\title{
Anti-inflammatory and Anti-tumorigenic Effects of Açai Berry in Helicobacter felis-infected mice
}

\author{
Ju Yup Lee ${ }^{1,3}$, Nayoung Kim¹, Yoon Jeong Choi ${ }^{1}$, Ryoung Hee Nam ${ }^{1}$, Seonmin Lee ${ }^{1}$, Min Hee Ham ${ }^{1}$, Ji Hyung Suh ${ }^{1}$, \\ Yoon Jin Choi ${ }^{1}$, Hye Seung Lee ${ }^{2}$, Dong Ho Lee ${ }^{1}$ \\ Departments of ${ }^{1}$ Internal Medicine and ${ }^{2}$ Pathology, Seoul National University Bundang Hospital, Seongnam, ${ }^{3}$ Department of Internal Medicine, \\ Keimyung University School of Medicine, Daegu, Korea
}

\begin{abstract}
Background: The aim of this study was to evaluate the anti-inflammatory and anti-tumorigenic effect of açai berry after chronic Helicobacter felis colonization in the stomachs of C57BL/6 mice.

Methods: A total of 57 four-week-old female C57BL/6 mice (18 control mice and 39 experimental mice) were used. The mice were administered orogastrically with vehicle only or vehicle containing $H$. felis, 5 times every other day. After inoculation of $H$. felis, mice were fed either a standard or an açai-containing diet and then sacrificed at 4, 24, and 52 weeks. The infection status and degree of inflammation were determined by culture and histopathology. The level of gastric mucosal myeloperoxidase (MPO), TNF- $\alpha$, and interleukin-1 $\beta$ (IL-1 $\beta$ ) were measured by ELISA.

Results: At 24 weeks after inoculation, mucosal atrophy and mucous metaplasia appeared in all infected mice. At 52 weeks after inoculation, dysplastic change was noted in $10 \%, 25 \%$, and $50 \%$ of mice in the $H$. felis-control, $H$. felis-açai $5 \%$, and $H$. felis-açai $10 \%$ groups, respectively. The neutrophil, monocyte, atrophy, and metaplasia grades of infected mice showed no significant difference among the $H$. felis-infected groups. $H$. felis-infected mice fed with açai berry showed no significant difference compared with $H$. felis-infected control mice in gastric mucosal MPO, TNF- $\alpha$, and IL-1 $\beta$ levels.

Conclusions: $H$. felis that colonized the stomachs of $\mathrm{C} 57 \mathrm{BL} / 6$ mice provoked inflammation, and induced mucosal atrophy, metaplasia, and dysplasia. However, açai berry did not effectively prohibit the gastric carcinogenesis which was induced by chronic $H$. felis infection. (J Cancer Prev 2016;21:48-54)
\end{abstract}

Key Words: Açai (Euterpe oleracea), Stomach, Helicobacter felis, Inflammation

\section{INTRODUCTION}

The prevalence of gastric cancer is high in Korea $^{1}$ and Helicobacter pylori infection is a major etiologic factor associated with gastric cancer, ${ }^{2}$ which shows a high prevalence of $54.4 \%$ among asymptomatic Korean adults. ${ }^{3} \mathrm{H}$. felis, which is analogous to $H$. pylori in humans, is one of the most common organisms colonizing the mouse stomach ${ }^{4}$ and causes natural infections in mice. ${ }^{5}$ Chronic $H$. felis infection induces severe inflammation, atrophy, metaplasia, dysplasia, and gastric cancer in $\mathrm{C} 57 \mathrm{BL} / 6$ mice. ${ }^{6}$ We previously demonstrated that $H$. felis colonized the stomachs of C57BL/6 mice and provoked severe inflammation, eventually inducing mucosal atrophy, metaplasia, dysplasia, and cancer. ${ }^{7}$ Numerous studies have focused on gastric cancer chemoprevention; among these approaches, blocking the $H$. pylori-associated gastric cancer pathway has been shown to be important for gastric cancer prevention.

Fruits and vegetables that contain various compounds, including antioxidant and anti-inflammatory compounds, are considered promising sources for preventive agents of various cancers. Among them, açai berry has received considerable attention in recent years as a 'super fruit' because of its high antioxidant capacity and potential anti-inflammatory activities. ${ }^{8}$ Açai (Euterpe oleracea Mart.) belongs to the family Arecaceae

Received February 29, 2016, Revised March 5, 2016, Accepted March 7, 2016

Correspondence to: Nayoung Kim

Department of Internal Medicine, Seoul National University Bundang Hospital, 82 Gumi-ro 173beon-gil, Bundang-gu, Seongnam 13620, Korea Tel: +82-31-787-7008, Fax: +82-31-787-4051, E-mail: nayoungkim49@empas.com, ORCID: Nayoung Kim, http://orcid.org/0000-0002-9397-0406

Copyright (C) 2016 Korean Society of Cancer Prevention

(c) This is an Open Access article distributed under the terms of the Creative Commons Attribution Non-Commercial License (http://creativecommons.org/licenses/by-nc/4.0) which permits unrestricted non-commercial use, distribution, and reproduction in any medium, provided the original work is properly cited. 
(palm tree) and is widely cultivated in the Amazon region of Brazil. It contains various essential nutrients, including fatty acids, amino acids, minerals, and antioxidant such as anthocyanins, proanthocyanidins, and other flavonoids. ${ }^{9}$ Açai was found to attenuate cell proliferation and tumor growth in an esophageal cancer rodent model, ${ }^{10}$ and açai feeding attenuated dimethylhydrazine-induced rat colon carcinogenesis. ${ }^{11}$ The açai flavonoid velutin is a potent anti-inflammatory agent that blocks lipopolysaccharide-mediated TNF- $\alpha$ and interleukin (IL)-6 production by inhibiting NF- $\mathrm{KB}$ activation and the mitogenactivated protein kinase pathway. ${ }^{12}$

Although consumption of açai has increased, few studies have examined its protective effect in gastric carcinogenesis. Thus, the aim of this study was to evaluate the anti-inflammatory and anti-tumorigenic effect of açai berry after chronic $H$. felis colonization in the stomachs of C57BL/6 mice.

\section{MATERIALS AND METHODS}

\section{Animals and Helicobacter felis infection}

A total of 57 (18 control mice and 39 experimental mice) four-week-old female C57BL/6 mice (Orient Co. Ltd., Seoul, Korea) weighing 10 to $15 \mathrm{~g}$ were used for the experiment.

The mice were administered orogastrically with vehicle only $\left(0.25 \mathrm{~mL}\right.$, for controls) or vehicle containing more than $1 \times 10^{7}$ colony- forming units/mL of $H$. felis (ATCC 49179), 5 times every other day. After inoculation of $H$. felis, mice were fed with a standard or an açai-containing diet for 4, 24, and 52 weeks, respectively (Fig. 1). Both the control and H. felis-infected groups were divided into 3 groups, respectively, according to the diet and housed in a cage maintained at $23^{\circ} \mathrm{C}$ with a $12 / 12$-hour light/dark cycle under specific pathogen-free conditions. Group 1 (con) included control mice fed with the standard diet; group 2 (açai 5\%) included control mice fed with $5 \%$ açai powder (AP); group 3 (açai $10 \%$ ) included control mice fed with $10 \%$ AP; group 4 (H. felis [HF]-con) included $H$. felis-infected mice fed with the standard diet; group 5 (HF-açai 5\%) included $H$. felis-infected mice fed with $5 \%$ AP; and group 6 (HF-açai 10\%) included $H$. felis-infected mice fed with $10 \% \mathrm{AP}$. The mice were sacrificed by $\mathrm{CO}_{2}$ asphyxiation at 4, 24, and 52 weeks after $H$. felis inoculation.

Açai berries were collected in Belem, Brazil and spray-dried using an industrial spray-dryer system with maltodextrin DE10 as a carrier agent. ${ }^{13} \mathrm{AP}$ was produced by Centroflora Group Brazil (Botucatu, Brazil) with the following characteristics: 6\% moisture, 350 to $650 \mathrm{~g} / \mathrm{L}$ volumetric density, and $0.5 \%$ total polyphenol content. ${ }^{13}$ Freeze-dried açai pulp powder was purchased from Boto Superfood Co. (Seoul, Korea), which imported the end product. Freeze-dried AP was stored at $-20^{\circ} \mathrm{C}$ until analysis. A cereal-based commercial diet containing $5 \%$ and $10 \%$ AP for mice was specially formulated by the Orient Bio (Seongnam, Korea) according to the National Research Council's recommendation to meet rodent nutritional needs. ${ }^{13}$ The standard diet group was fed with LabDiet for rodent (Orient Bio) which contained 18\% protein, $5.2 \%$ fat, and other crude fiber and minerals.

\section{Histopathology}

At necropsy, stomach tissue was taken from the greater curvature beginning at the squamocolumnar junction and ending at the gastroduodenal junction. Linear gastric strips were fixed in $10 \%$ formalin solution, processed using standard methods, embedded in paraffin, sectioned at $5 \mu \mathrm{m}$, and stained with hematoxylin and eosin. The stomach mucosa was histologically examined for inflammatory and epithelial changes and for the presence of $H$. felis. The degree of neutrophil infiltration, mononuclear cell infiltration, atrophy, and metaplasia were assessed according to the updated Sydney classification as follows: 0, absent; 1 , minimal; 2, mild; 3, moderate; 4, marked.

\section{Measurement of mucosal myeloperoxidase, TNF- $\alpha$, and interleukin-1 $\beta$}

Ten milligrams of scraped mucosa were homogenized for 30 seconds with a Polytron homogenizer in $200 \mu \mathrm{L}$ of ice-cold lysis

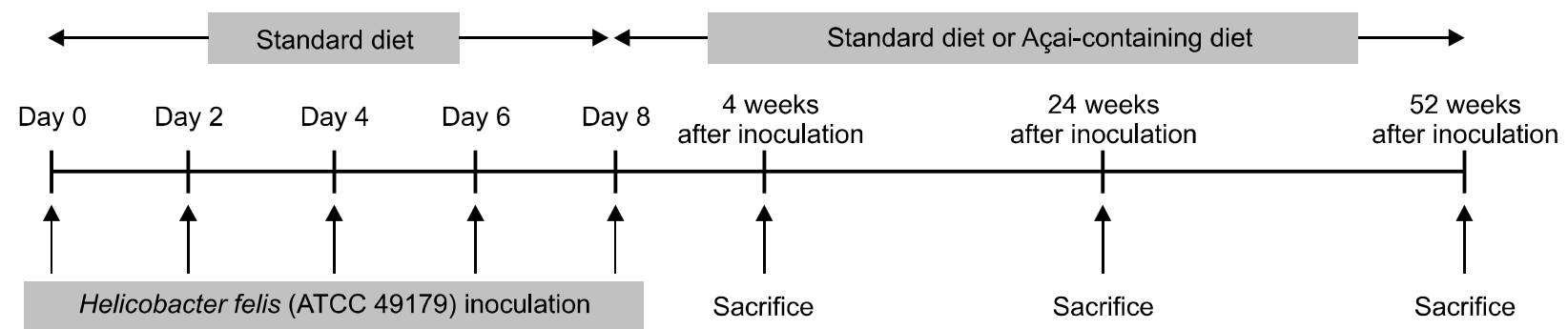

Figure 1. Schematic representation of the experimental design. 
buffer (200 mM NaCl, 5 mM EDTA, $10 \mathrm{mM}$ Tris [pH 7.4], 10\% glycerin, $1 \mathrm{mM}$ phenylmethylsulfonyl fluoride, $1 \mu \mathrm{g} / \mathrm{mL}$ leupeptin, and $28 \mu \mathrm{g} / \mathrm{mL}$ aprotinin). The cell suspensions were centrifuged at $13,000 \mathrm{rpm}$ for 15 minutes, and the resulting supernatant was assayed using a myeloperoxidase (MPO) ELISA kit (HyCult Biotechnology, Uden, The Netherlands). For TNF- $\alpha$ and IL-1 $\beta$, the appropriate kits from R\&D Systems (Minneapolis, MN, USA) were used following the manufacturer's instructions. Protein concentration was measured using a Bio-Rad Protein Assay Kit (Bio-Rad Laboratories, Hercules, CA, USA). The concentration of each cytokine was measured as $\mathrm{pg} / \mathrm{mg}$ of protein. All assays were performed in triplicate.

\section{Statistical analysis}

Data are expressed as the mean \pm SEM. Comparison between 2 groups (control and experimental) was performed using the Mann-Whitney U-test. $P$-values less than 0.05 were considered statistically significant. All statistical analyses were performed using IBM SPSS software ver. 20.0 (IBM Co., Armonk, NY, USA).

\section{RESULTS}

\section{Histopathology}

At 4 weeks after inoculation, neutrophil and monocyte infiltration occurred in all infected mice. At 24 weeks after inoculation, mucosal atrophy and mucous metaplasia appeared in most of infected mice. At 52 weeks after inoculation, dysplastic change was noted in 20\%, 25\%, and 50\% of mice in the $H$. felis-control, $H$. felis-açai 5\%, and H. felis-açai $10 \%$ groups, respectively. Gastric cancer was not observed in any infected mice (Table 1).

The neutrophil and monocyte grades of infected mice peaked at week 24 and were significantly higher compared with the control mice; however, there was no significant difference among the $H$. felis-control, $H$. felis-açai 5\%, and $H$. felis-açai $10 \%$ groups (Fig. 2A and 2B). No atrophy and metaplasia occurred up to week 4; however, all infected mice showed higher atrophic and metaplasia scores than control mice after 24 weeks. There was no significant difference among the $H$. felis-control, $H$. felis-açai 5\%, and H. felis-açai 10\% groups (Fig. 2C and 2D).

\section{Expression of pro-inflammatory cytokines}

The gastric mucosal MPO level (ng/mL) in $H$. felis-infected mice was significantly higher than in control mice at 24 and 52 weeks; however $H$. felis-infected mice fed with açai berry showed no significant difference compared with $H$. felis-infected control mice (Fig. 3A). Gastric mucosal TNF- $\alpha$ expression (pg/mL) in $H$. felis-infected mice showed no significant difference compared with control mice at 4, 24, and 52 weeks (Fig. 3B). Gastric mucosal IL-1 $\beta$ expression (pg/mL) in $H$. felis-infected mice was significantly higher than in control mice at 24 and 52 weeks; however $H$. felis-infected mice fed with açai berry showed no significant difference compared with $H$. felis-infected control mice (Fig. 3C).

\section{DISCUSSION}

In this study, we observed inflammation, atrophy, metaplasia, and dysplasia in mouse stomachs after $H$. felis inoculation; however, Açai berry did not effectively reduce inflammation and dysplasia.

It has been reported that in various inflammation models, açai berry extract has anti-inflammatory and antioxidant effects by regulating protein enzymes expressed by pro-inflammatory cytokines and induced by oxidative stress. Xie et al. ${ }^{12}$ showed that compounds in açai effectively inhibited the expression of TNF- $\alpha$ and IL- 6 by inhibiting NF-KB activation and p38 and JNK

Table 1. Histologic findings in mice infected with Helicobacter felis during 52 weeks

\begin{tabular}{|c|c|c|c|c|c|c|c|c|c|c|}
\hline \multirow{2}{*}{ Histologic finding } & \multirow{2}{*}{ Control } & \multicolumn{3}{|c|}{4 weeks } & \multicolumn{3}{|c|}{24 weeks } & \multicolumn{3}{|c|}{52 weeks } \\
\hline & & Standard & Açai 5\% & Açai $10 \%$ & Standard & Açai 5\% & Açai $10 \%$ & Standard & Açai 5\% & Açai $10 \%$ \\
\hline H. felis $(+)$ & $0 / 18$ & $6 / 6$ & $4 / 4$ & $4 / 4$ & $5 / 5$ & $3 / 3$ & $4 / 4$ & $5 / 5$ & $4 / 4$ & $3 / 4$ \\
\hline Neutrophil & $2 / 18$ & $3 / 6$ & $1 / 4$ & $2 / 4$ & $3 / 5$ & $3 / 3$ & $4 / 4$ & $4 / 5$ & $4 / 4$ & $3 / 4$ \\
\hline Monocyte & $2 / 18$ & $1 / 6$ & $1 / 4$ & $0 / 4$ & $5 / 5$ & $3 / 3$ & $4 / 4$ & $5 / 5$ & $4 / 4$ & $4 / 4$ \\
\hline Atrophy & $0 / 18$ & $0 / 6$ & $0 / 4$ & $0 / 4$ & $3 / 5$ & $3 / 3$ & $4 / 4$ & $3 / 5$ & $3 / 4$ & $3 / 4$ \\
\hline Metaplasia & $0 / 18$ & $0 / 6$ & $0 / 4$ & $0 / 4$ & $3 / 5$ & $3 / 3$ & $4 / 4$ & $3 / 5$ & $3 / 4$ & $3 / 4$ \\
\hline Lymphoid aggregate & $0 / 18$ & $0 / 6$ & $0 / 4$ & $0 / 4$ & $3 / 5$ & $3 / 3$ & $2 / 4$ & $4 / 5$ & $1 / 4$ & $3 / 4$ \\
\hline Dysplasia & $0 / 18$ & $0 / 6$ & $0 / 4$ & $0 / 4$ & $0 / 5$ & $0 / 3$ & $0 / 4$ & $1 / 5$ & $1 / 4$ & $2 / 4$ \\
\hline Gastric cancer & $0 / 18$ & $0 / 6$ & $0 / 4$ & $0 / 4$ & $0 / 5$ & $0 / 3$ & $0 / 4$ & $0 / 5$ & $0 / 4$ & $0 / 4$ \\
\hline
\end{tabular}

Values are presented as the number of mice with histological findings/total number of mice. Açai, açai berry. 
A

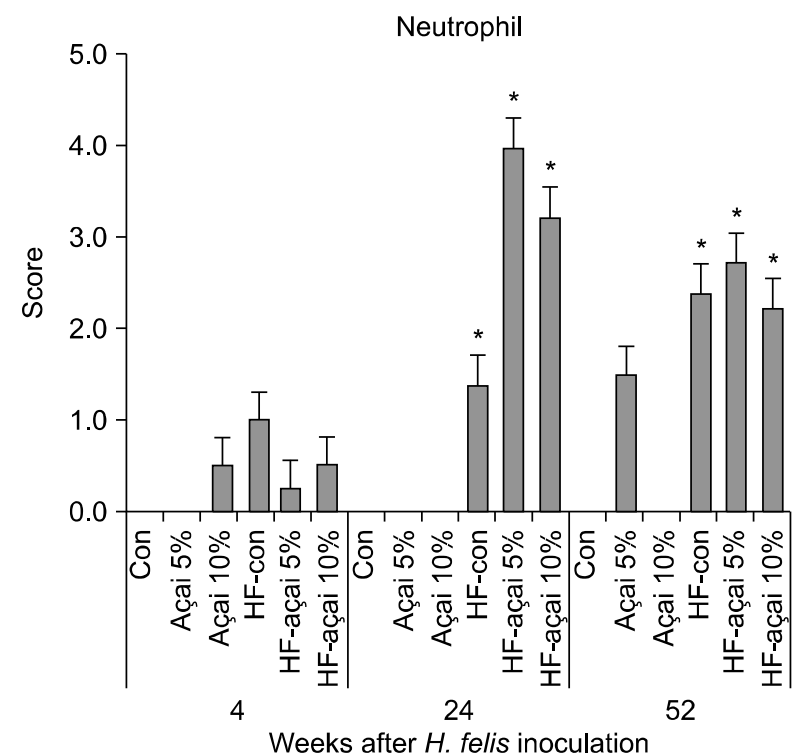

C

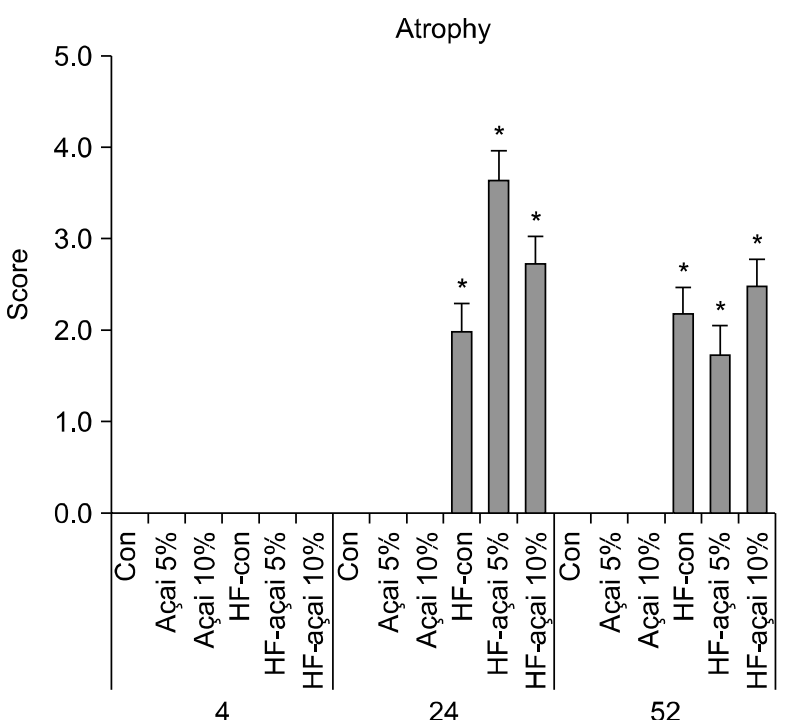

Weeks after $H$. felis inoculation
B

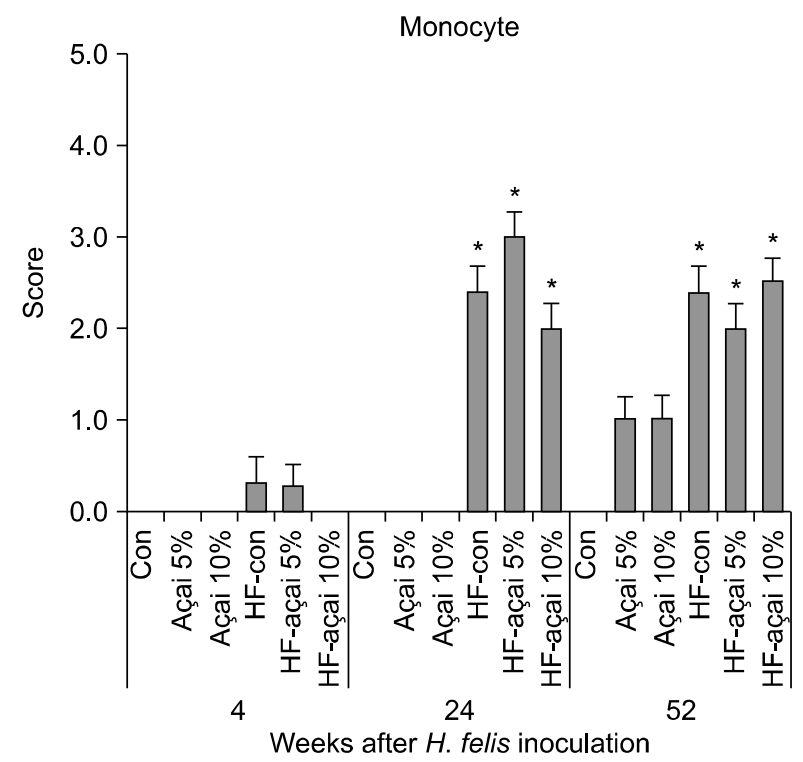

D

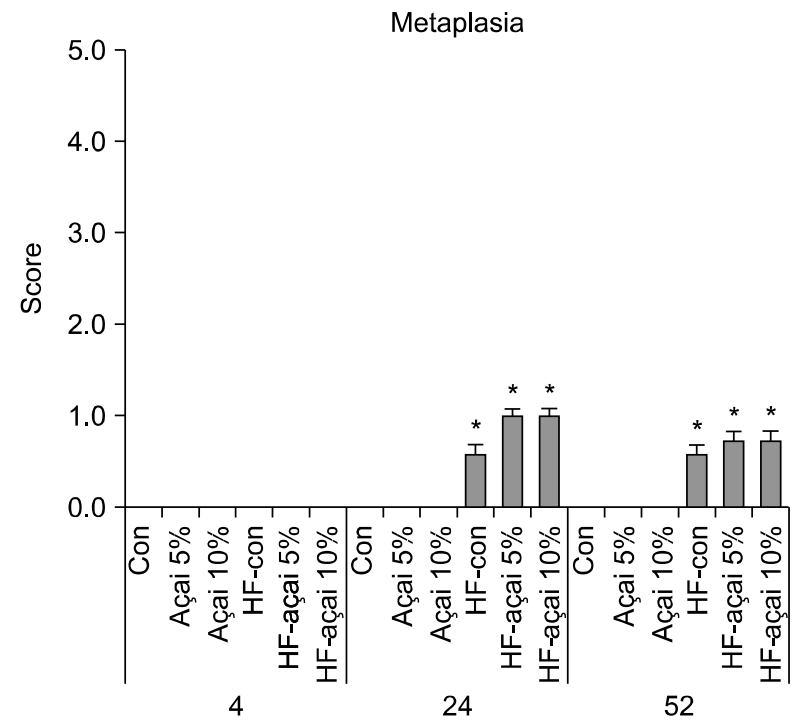

Weeks after $H$. felis inoculation

Figure 2. Gastric histopathology scores of (A) neutrophil, (B) monocyte, (C) atrophy, and (D) metaplasia at 4, 24, and 52 weeks after Helicobacter felis $(\mathrm{HF})$ inoculation and standard diet or diets with açai powder. Data are presented as means \pm SEMs. $* P<0.05$ compared with controls of the same diet. Con, control.

phosphorylation. They also found lower serum levels of TNF- $\alpha$ and IL- 6 in apoE-deficient mice fed with $5 \%$ freeze-dried açai juice powder for 20 weeks. In a mouse experiment, açai extract also reduced inflammatory and oxidant markers such as MPO, superoxide dismutase, catalase, gluthathione peroxidase, TNF- $\alpha$, and nitrites that increase by cigarette smoking. ${ }^{14}$

Açai berry also has anticancer activities. Açai berry effectively inhibited N-nitrosomethylbenzylamine-induced esophageal cancer and reduced serum levels of IL-5 and IL-8, which may have an inhibitory role in F344 rats. ${ }^{15}$ Açai berry upregulated serum levels of IFN- $\gamma$ and activated macrophage-released IFN- $\gamma$ induced apoptosis through the Fas/FasL pathway in glioma cells. ${ }^{16}$ In addition, many berry types may function through a tumor inhibition mechanism in nitrosomethylbenzylamine-treated rat esophagus. ${ }^{15}$ Açai also reduced the development of dimethylhydrazine-induced rat colon carcinogenesis. ${ }^{11}$

It has been shown that berry compounds, including cranberry, raspberry, and strawberry extracts, are capable of preventing the 
A

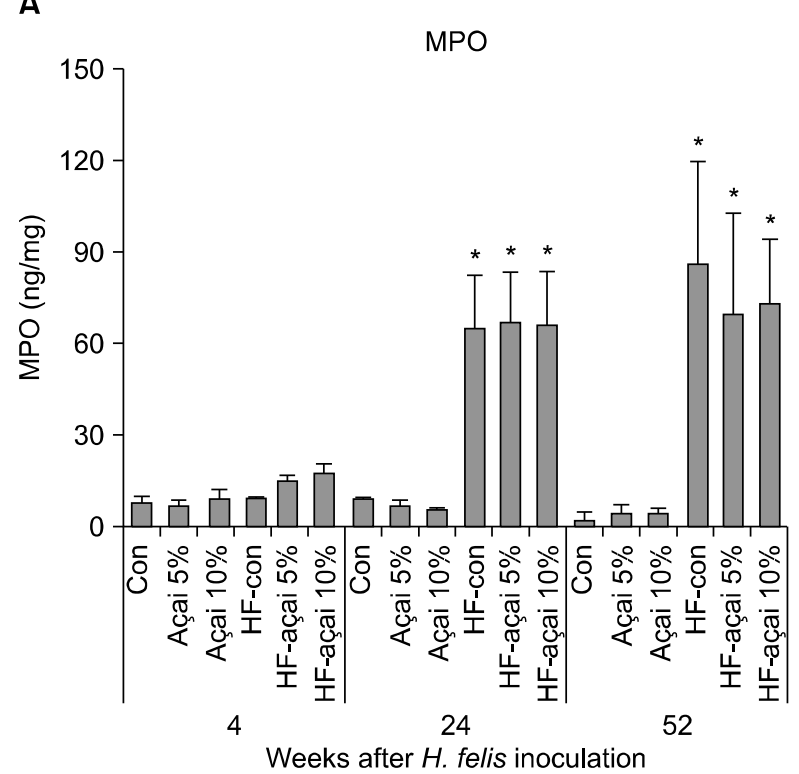

C

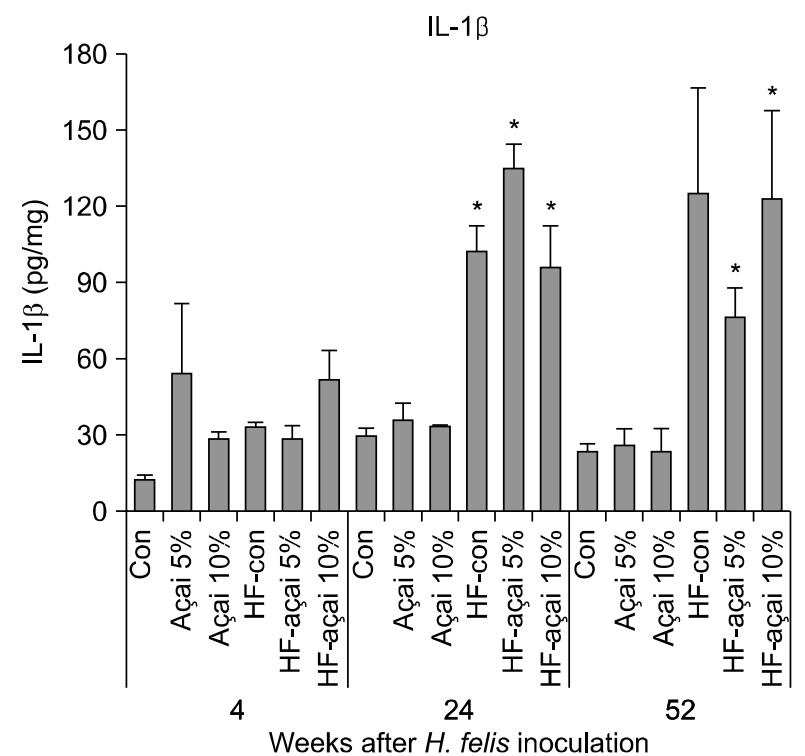

adhesion of $H$. pylori to gastric mucosa cells. ${ }^{17}$ High-molecularweight compounds such as proanthocyanidins isolated from cranberry extracts can interfere in vitro with some adhesins of $H$. pylori, inhibiting its adhesion to the human gastric mucosa. ${ }^{18,19}$ In vitro experiments involving cultured $H$. pylori showed that various berry extracts (raspberry, strawberry, cranberry, elderberry, blueberry, and bilberry) significantly inhibited H. pylori compared with controls and increased the susceptibility of $H$. pylori to clarithromycin. ${ }^{20}$ OptiBerry, prepared from a blend of the above 6 extracts, showed the largest effect. ${ }^{20}$ A Chinese randomized,
B

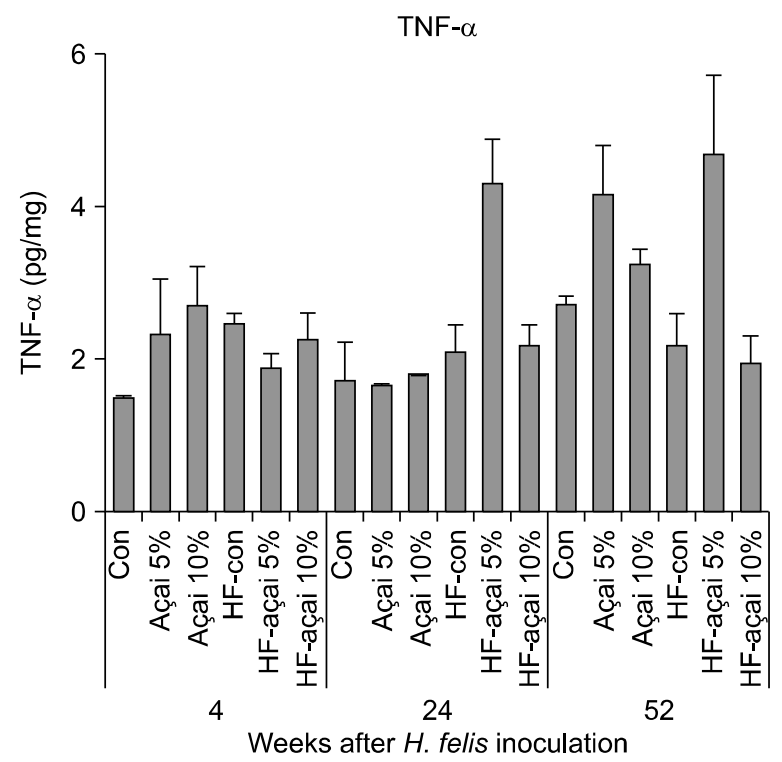

Figure 3. Expression of gastric mucosal (A) myeloperoxidase (MPO), (B) TNF- $\alpha$, and (C) interleukin (IL)- $1 \beta$ by ELISA at 4,24 , and 52 weeks after Helicobacter felis (HF) inoculation and standard diet or diets with açai powder. Data are presented as means \pm SEMs. $* P<0.05$ compared with controls of the same diet. Con, control.

double blind, placebo-controlled trial demonstrated that regular cranberry juice consumption suppressed $H$. pylori infection. ${ }^{21}$

Few studies have examined the effectiveness of açai berry in suppressing $H$. pylori infection. However, similar to cranberry, açai berry contains proanthocyanidins and we expected that açai also inhibit $H$. felis via the same mechanism of cranberry. However, our experiment showed negative result. We used $H$. felis instead of $H$. pylori because in our previous study $H$. felis provoked more severe inflammation and dysplasia than $H$. pylori. $^{7} \mathrm{H}$. felis shows noticeably different pathological features 
compared with the pathophysiological changes occurring in human gastric mucosa. Moreover, H. felis lacks the cag pathogenicity island and does not adhere well to gastric epithelial cells, floating in the gastric mucosa. ${ }^{22}$ Unlike $H$. felis that has weak adhesion or float in the mucus when observed with an electron microscope, $H$. pylori is characterized by tight adhesion to the epithelial cells via fine projections with adherence pedestals. ${ }^{22}$ Therefore, it is possible that proanthocyanidins act to H. felis in a different way. Another limited point of this study is the concentration of açai berry. We used $5 \%$ and $10 \%$ of AP based on our preliminary study in colon (data not shown) which showed limited effects at $2.5 \%$ of açai berry. We expected more effective result on $10 \%$ of açai berry. However, in our study, the dysplastic change was stepwise increased from no açai berry to $10 \%$ açai berry group. Although stomach and colon is different organ, $10 \%$ of açai berry concentration was higher than those of previous carcinogenesis studies treated with 2.5\% and 5\%. ${ }^{11,15}$ There was no study about the optimal concentration of açai berry for prevention of gastric carcinogenesis, however, it is possible that $10 \%$ concentration of açai berry is too high and may induce adverse effects in $H$. felis mouse model.

In conclusion, $H$. felis that colonized the stomachs of C57BL/6 mice provoked inflammation, mucosal atrophy, metaplasia, and dysplasia. Açai berry did not effectively inhibit the gastric carcinogenesis which was induced by chronic $H$. felis infection. Further studies are required to elucidate the mechanism underlying anti-inflammatory and anti-tumorigenic effect of açai berry in mouse and human stomach.

\section{ACKNOWLEDGMENTS}

This work was supported by the National Research Foundation of Korea (NRF) grant for the Global Core Research Center (GCRC) funded by the Korea government (MSIP) (No. 2011-0030001).

\section{CONFLICTS OF INTEREST}

No potential conflicts of interest were disclosed.

\section{REFERENCES}

1. Jung KW, Won YJ, Kong HJ, Oh CM, Cho H, Lee DH, et al. Cancer statistics in Korea: incidence, mortality, survival, and prevalence in 2012. Cancer Res Treat 2015;47:127-41.

2. McColl KE. Clinical practice. Helicobacter pylori infection. N Engl J Med 2010;362:1597-604.

3. Lim SH, Kwon JW, Kim N, Kim GH, Kang JM, Park MJ, et al. Prevalence and risk factors of Helicobacter pylori infection in
Korea: nationwide multicenter study over 13 years. BMC Gastroenterol 2013;13:104.

4. Hayakawa Y, Fox JG, Gonda T, Worthley DL, Muthupalani S, Wang TC. Mouse models of gastric cancer. Cancers (Basel) 2013:5:92-130.

5. Lee A, Hazell SL, O'Rourke J, Kouprach S. Isolation of a spiral-shaped bacterium from the cat stomach. Infect Immun 1988;56:2843-50.

6. Fox JG, Sheppard BJ, Dangler CA, Whary MT, Ihrig M, Wang TC. Germ-line p53-targeted disruption inhibits helicobacter-induced premalignant lesions and invasive gastric carcinoma through down-regulation of Th1 proinflammatory responses. Cancer Res 2002;62:696-702.

7. Lee JY, Kim N, Choi YJ, Nam RH, Choi YJ, Kwon YH, et al. Histologic Findings and Inflammatory Reactions After Long-term Colonization of Helicobacter felis in C57BL/6 Mice. J Cancer Prev 2014;19:224-30

8. Kang J, Xie C, Li Z, Nagarajan S, Schauss AG, Wu T, et al. Flavonoids from acai (Euterpe oleracea Mart.) pulp and their antioxidant and anti-inflammatory activities. Food Chem 2011;128: 152-7.

9. Schauss AG, Wu X, Prior RL, Ou B, Patel D, Huang D, et al. Phytochemical and nutrient composition of the freeze-dried amazonian palm berry, Euterpe oleraceae mart. (acai). J Agric Food Chem 2006:54:8598-603.

10. Stoner GD. Foodstuffs for preventing cancer: the preclinical and clinical development of berries. Cancer Prev Res (Phila) 2009;2: $187-94$.

11. Fragoso MF, Romualdo GR, Ribeiro DA, Barbisan LF. Açai (Euterpe oleracea Mart.) feeding attenuates dimethylhydrazine-induced rat colon carcinogenesis. Food Chem Toxicol 2013;58:68-76.

12. Xie C, Kang J, Li Z, Schauss AG, Badger TM, Nagarajan S, et al. The açaí flavonoid velutin is a potent anti-inflammatory agent: blockade of LPS-mediated TNF- $\alpha$ and IL-6 production through inhibiting NF- $\kappa \mathrm{B}$ activation and MAPK pathway. J Nutr Biochem 2012;23:1184-91.

13. National Research Council. Nutrient requirements of the laboratory rat. In: National Research Council, ed. Nutrient Requirements of Laboratory Animals. 4th ed. Washington, DC, National Academy Press, pp 11-79, 1955.

14. Moura RS, Ferreira TS, Lopes AA, Pires KM, Nesi RT, Resende AC, et al. Effects of Euterpe oleracea Mart. (AÇAÍ) extract in acute lung inflammation induced by cigarette smoke in the mouse. Phytomedicine 2012;19:262-9.

15. Stoner GD, Wang LS, Seguin C, Rocha C, Stoner K, Chiu S, et al. Multiple berry types prevent N-nitrosomethylbenzylamine-induced esophageal cancer in rats. Pharm Res 2010;27:1138-45.

16. Chen GG, Chu YS, Chak EC, Leung BC, Poon WS. Induction of apoptosis in glioma cells by molecules released from activated macrophages. J Neurooncol 2002;57:179-86.

17. Folmer F, Basavaraju U, Jaspars M, Hold G, El-Omar E, Dicato M, et al. Anticancer effects of bioactive berry compounds. Phytochem Rev 2014:13:295-322.

18. Shmuely H, Burger O, Neeman I, Yahav J, Samra Z, Niv Y, et al. Susceptibility of Helicobacter pylori isolates to the antiadhesion activity of a high-molecular-weight constituent of cranberry. Diagn Microbiol Infect Dis 2004:50:231-5.

19. Gotteland M, Andrews M, Toledo M, Muñoz L, Caceres P, Anziani A, et al. Modulation of Helicobacter pylori colonization with cran- 
berry juice and Lactobacillus johnsonii Lal in children. Nutrition 2008:24:421-6.

20. Chatterjee A, Yasmin T, Bagchi D, Stohs SJ. Inhibition of Helicobacter pylori in vitro by various berry extracts, with enhanced susceptibility to clarithromycin. Mol Cell Biochem 2004:265:19-26.

21. Zhang L, Ma J, Pan K, Go VL, Chen J, You WC. Efficacy of cran- berry juice on Helicobacter pylori infection: a double-blind, randomized placebo-controlled trial. Helicobacter 2005; 10:139-45.

22. Lee A, O'Rourke J, De Ungria MC, Robertson B, Daskalopoulos G, Dixon MF. A standardized mouse model of Helicobacter pylori infection: introducing the Sydney strain. Gastroenterology 1997:112:1386-97. 